\title{
All-optical NRZ-to-RZ reconversion from the red-chirped NRZ signal generated by the RZ-to-NRZ converter using an SOA-loop-mirror
}

\author{
Hyuek Jae Lee ${ }^{\text {a) }}$ \\ Division of Information \& Communication Engineering, Kyungnam University, \\ 449 Wolyoung-dong, Changwon, 631-701, KOREA \\ a)hyuek@kyungnam.ac.kr
}

\begin{abstract}
This paper aims to demonstrate experimentally a new all-optical non-return-to-zero (NRZ) to return-to-zero (RZ) data format reconversion from the red-chirped NRZ signal, generated by the RZ-to-NRZ converter, using a semiconductor optical amplifier in a fiber loop mirror (so-called an SOA-loop-mirror). The primary merit of the proposed NRZ-to-RZ reconversion is that no additional optical clock is required in the process, unlike other conventional methods. While the optical power penalty for the RZ-to-NRZ conversion using an SOA-loop-mirror was $1.64 \mathrm{~dB}$, the proposed NRZ-to-RZ reconversion penalty was $0.6 \mathrm{~dB}$ compared to that of the original input $\mathrm{RZ}$ signal.
\end{abstract}

Keywords: all-optical data format conversion, non-return-to-zero (NRZ), return-to-zero (RZ), SOA-loop-mirror, wavelength division multiplexing (WDM), optical time division multiplexing (OTDM)

Classification: Fiber optics, Microwave photonics, Optical interconnection, Photonic signal processing, Photonic integration and systems

\section{References}

[1] D. Norte, E. Park and A. E. Willner: IEEE Photon. Technol. Lett. 7 [8] (1995) 920.

[2] D. Norte and A. E. Willner: J. Lightw. Technol. 14 [6] (1996) 1170.

[3] B. Mikkelsen, M. Vaa, H. N. Poulsen, S. L. Danielsen, C. Joergensen, A. Kloch, P. B. Hansen, K. E. Stubkjaer, K. Wunstel, K. Daub, E. Lach, G. Laube, W. Idler, M. Schilling and S. Bouchoule: Electron. Lett. 33 (1997) 133.

[4] P. S. Cho, D. Mahgerefteh and J. Goldhar: Proc. Eur. Conf. Optical Communication (ECOC1998) (1998) 353.

[5] H. J. Lee, S. J. B. Yoo and C.-S. Park: Optical Fiber Communication Conf. (OFC 2001) (2001) MB7.

[6] C. G. Lee, Y. J. Kim, C. S. Park, H. J. Lee and C. S. Park: J. Lightw. Technol. 23 [2] (2005) 834. 
[7] H. J. Lee, K. Kim, J. Y. Choi, H. G. Kim and C. H. Yim: IEICE Trans. Commun. E82-B (1999) 387.

[8] L. Xu, B. C. Wang, V. Baby, I. Glesk and P. R. Prucnal: Opt. Commun. 206 (2002) 77.

[9] S. G. Park, L. H. Spiekman, M. Eiselt and J. M. Wiesenfeld: IEEE Photon. Technol. Lett. 12 [3] (2000) 233.

[10] C. W. Chow, C. S. Wong and H. K. Tsang: Opt. Commun. 209 (2002) 329.

[11] K. Mishina, A. Maruta, S. Mitani, T. Miyahara, K. Ishida, K. Shimizu, T. Hatta, K. Motoshima and K. Kitayama: J. Lightw. Technol. 24 (2006) 3751.

[12] K. Mishina, T. Kono, A. Maruta and K. Kitayama: Opto-Electronics and Communications Conference (OECC2012, 17th) (2012) 337.

[13] M. Eiselt, W. Pieper and H. G. Weber: J. Lightw. Technol. 13 [10] (1995) 2099.

[14] T. Inoue and S. Namiki: Proc. Eur. Conf. Optical Communication (ECOC2005) 1 (2005) Mo3.5.2.

[15] A. Shen, J. Damon-Lacoste, M. Le Pallec, C. Duchet, J. Gentner, F. Devaux and M. Renaud: Optical Fiber Communication Conf. (OFC) (2002) 339-WV3.

[16] P. P. Baveja, D. N. Maywar, A. M. Kaplan and G. P. Agrawal: IEEE J. Quantum Electron. 46 [9] (2010) 1396.

[17] H. J. Lee and H. G. Kim: IEEE Photon. Technol. Lett. 11 [4] (1999) 469.

\section{Introduction}

Wavelength-division multiplexing (WDM) and optical-time-division multiplexing (OTDM) technologies are the two promising techniques which may be the basis for the construction of future all-optical networks $[1,2]$. These two approaches would essentially use two standard data formats, viz. return-to-zero (RZ) and non-return-to-zero (NRZ). Generally, the RZ format relies on bit-interleaving method and is preferred in ultra-fast OTDM networks. The NRZ format, on the other hand, has a lower bandwidth requirement and a higher timing jitter tolerance than RZ format. Therefore, a conversion between the two data formats becomes necessary in linking and interfacing ultrafast OTDM networks and lowspeed WDM networks [1,2]. Additionally, in order to cascade several such networks while maintaining an optical transparency, a format reconversion to the original data $(\mathrm{RZ} \rightarrow \mathrm{NRZ} \rightarrow \mathrm{RZ}$ in this paper) is also important.

An appreciable number of all-optical data format conversions have been reported to date, using semiconductor optical amplifier (SOA) gain compression [1, 2], a monolithically integrated Michelson interferometer (MI) employing SOAs [3], an SOA/fiber grating wavelength converter [4], a nonlinear optical loop mirror (NOLM) with an SOA $[5,6,7]$, cross-phase modulation (XPM) in an integrated Mach-Zehnder interferometer (MZI) employing SOAs [8, 9], and Fabry-Perot (FP) laser diode with dualwavelength injection locking [10]. Currently, research have also been extended to all-optical format conversions between on-off keying (OOK) and phase-shift keying (PSK) [11, 12]. 
In this paper, a new all-optical NRZ-to-RZ reconversion has been proposed and experimentally demonstrated, which is cascaded with the previously proposed RZ-to-NRZ converter $[5,6]$ using an SOA in a fiber loop mirror (i.e. an SOA-loop-mirror). Conventional NRZ-to-RZ conversions $[1,2]$ are commonly performed through optical AND logic operations for an incoming NRZ signal and an optical clock is always necessary in such an approach. On the contrary, the proposed NRZ-to-RZ reconversion method requires only NRZ data signal, bypassing the use of any optical clock. This is possible because an NRZ signal contains the original RZ signal components by optical chirping.

\section{Principle}

The dashed box 'A' in Fig. 1 shows the schematic diagram of a RZ-to-NRZ converter obtained from prior literature $[5,6]$. The conversion method was based on a nonlinear optical loop mirror using an SOA (i.e. an SOA-loopmirror), which is often used in all-optical switching in OTDM networks [13]. However, the principle here is quite different. The terms $x(t)$ and $y(t)$ denote the electric fields of the input and the output data signals for the RZ-to-NRZ converter, respectively. The input RZ signal $x(t)$ in Fig 1 enters the fiber loop through the WDM coupler1. Because the SOA is located at the displacement of $\tau / 2(\tau \leq T, T$ is a signal period) from the mid point of the fiber loop, the $c c w(t)$ (counter clockwise) beam takes a phase changing effect later $\tau$ than the $c w(t)$ (clockwise) beam as shown in Fig. 2 (c). Strictly speaking, at the rising time of the input RZ signal $x(t)$, not alone phase change but also optical red-chirping are induced from the refractive-index change in the SOA active medium. As a result, the $c w(t)$

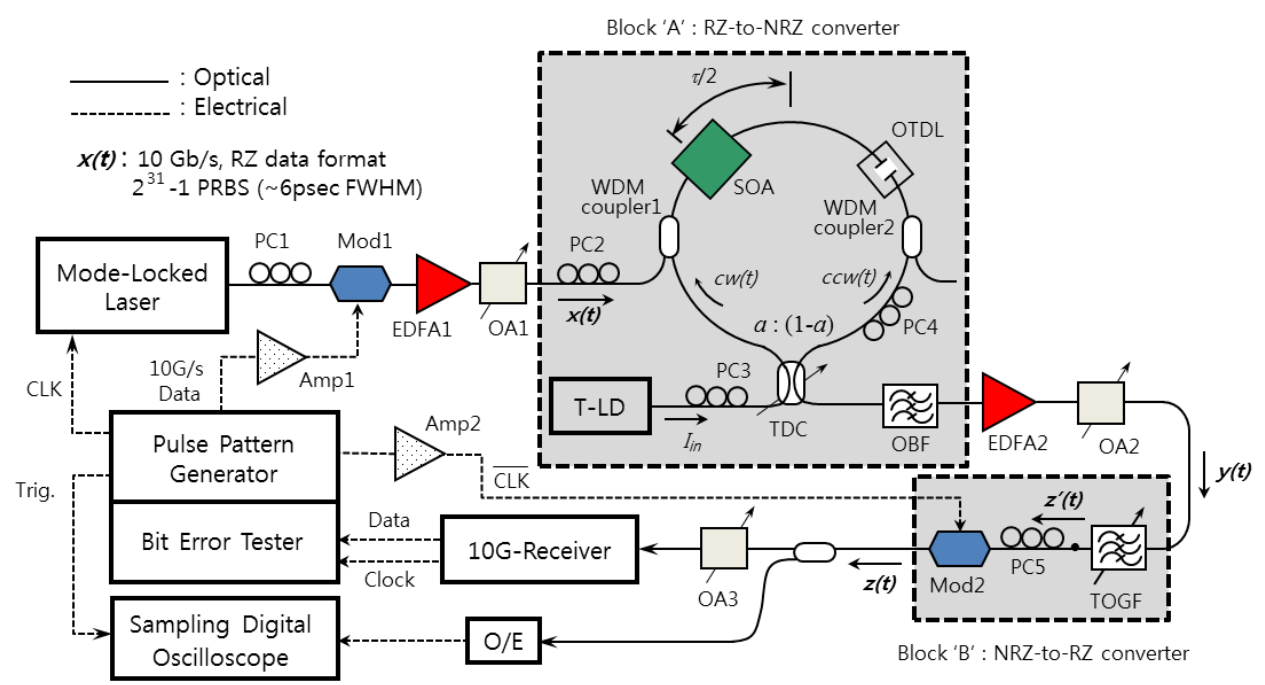

Fig. 1. Experimental setup for testing the proposed NRZto-RZ reconversion. (PC: Polarization Controller, EDFA: Er-doped Fiber Amplifier, OA: Optical Attenuator, SOA: Semiconductor Optical Amplifier, T-LD: Tunable Laser Diode, TDC: Tunable Directional Coupler, OTDL: Optical Tunable Delay Line, OTGF: Optical Tunable Grating Filter, OBF: Optical Bandpass Filter, Mod: Modulator). 
(a)

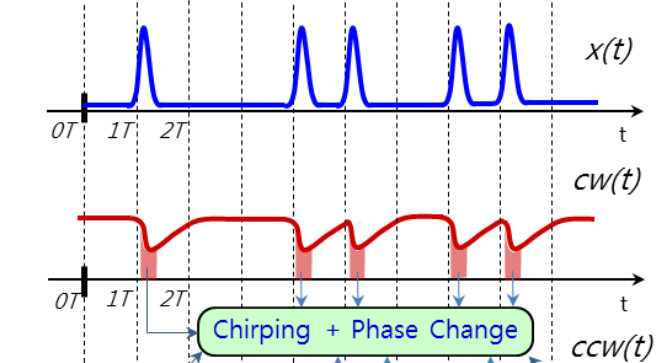

(c)

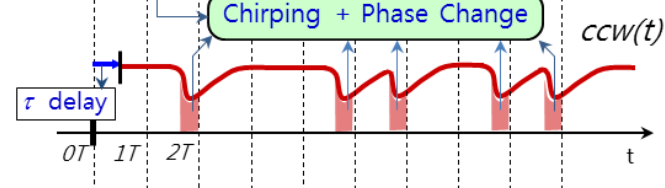

(d)

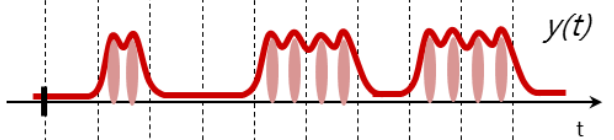

(e)

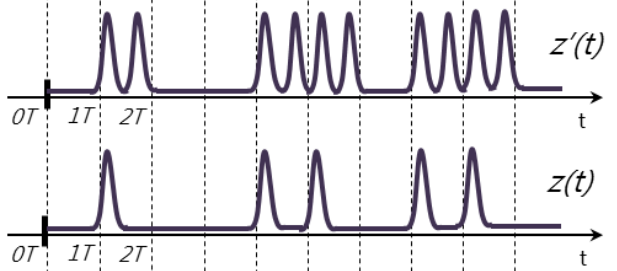

Fig. 2. Operational principle of the NRZ-to-RZ format reconversion from the red-chirped NRZ signal generated by the previously proposed RZ-to-NRZ converter $[5,6]$.

and $c c w(t+\tau)$ are gain-shaped to balance the relative phase changes (Fig. 2 (b) and (c)) [6]. If $\tau$ is adjusted to be less than $T$, the output $y(t)$ of the SOA-loop mirror shows the NRZ-like pulse pattern as Fig. 2 (d).

This NRZ pulse has red-chirped components at both the rising and falling edge. Owing to the red-chirpings, the converted NRZ signal has good transmission performances $[5,6]$.

The proposed NRZ-to-RZ converter is shown in the dashed block ' $\mathrm{B}$ ' of Fig. 1, which uses a sharp filtering to extract the two red-chirped components contained in the NRZ signal $y(t)$. The grating filter (TOGF) pulls out the two components as RZ signal, one of which is removed by the optical Mach-Zehnder modulator (Mod2). Unlike the conventional methods $[1,2]$, the proposed NRZ-to-RZ conversion has a merit of not requiring any extra optical clock owing to containing RZ signal components in the input NRZ signal $y(t)$. Here, to remove one of the two pulses, the electrical clock from the pulse pattern generator $(\mathrm{PPG})$ is used as the input to the optical Mach-Zehnder modulator (Mod2) as shown in Fig. 1. Otherwise, the clock should be made from the NRZ signal $y(t)$ or $z^{\prime}(t)$ by an appropriate clock recovery circuit even though the circuit is not shown in Fig. 1. From a different point of view, we might use an optical MachZehnder modulator to directly make RZ pulse $z(t)$ from input NRZ signal $y(t)$. However, it requires a RZ-like electrical pulse with an ultra-high operating speed (depends on the speed of the OTDM network).

\section{Experimental setup}

Fig. 1 shows the experimental setup, where the dashed boxes ' $\mathrm{A}$ ' and 'B' 
depict a RZ-to-NRZ and an NRZ-to-RZ converters, respectively. The schematic diagram of the NRZ-to-RZ converter proposed in this paper is the box 'B'. For a RZ-to-NRZ conversion, the SOA-loop-mirror previously developed $[5,6]$ is used, which consists of a TDC, an OTDL, a PC4, and an SOA. On the other hand, the proposed NRZ-to-RZ converter is simply composed of a tunable optical grating filter (TOGF), a PC5 and a LiNbO3 optical Mach-Zehnder modulator (Mod2). A LiNbO3 optical Mach-Zehnder modulator (Mod1) and a mode-locked laser ( $\sim 6 \mathrm{ps}$ full-width at halfmaximum (FWHM) at $10 \mathrm{GHz}$ repetition rate) driven by the pulse pattern generator (PPG) generates a $10 \mathrm{~Gb} / \mathrm{s} 2^{31}-1$ pseudo-random bit sequence (PRBS) data stream of RZ format at $1557.13 \mathrm{~nm}$. The RZ input signal $x(t)$ is fed into the SOA-loop-mirror through the WDM coupler1. For NRZ data generation, the continuous wave beam $I_{\text {in }}$ at $1550 \mathrm{~nm}$, generated from the tunable laser diode (T-LD), enters into one arm of the SOA-loop-mirror as shown in Fig. 1. The SOA used in this experiment is $1000 \mu \mathrm{m}$ long and nearly polarization insensitive (typically $\sim 0.6 \mathrm{~dB}$ ). The SOA-arrival time difference $\tau$ was set to $\sim 70$ psec. The SOA current was $190 \mathrm{~mA}$ and the coupling coefficient $\alpha$ of the TDC was adjusted to 0.41 .

\section{Results and discussions}

Fig. 3 (a) and (b) show the eye diagrams of the $10 \mathrm{~Gb} / \mathrm{s}$ input RZ signal and the RZ-to-NRZ converted signal, respectively. Previous studies $[5,6]$

(a)

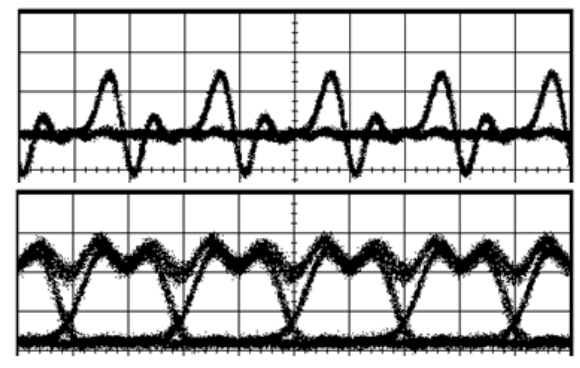

(b)

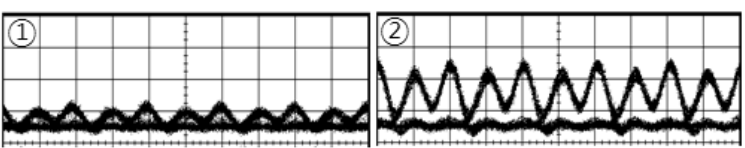

(c)
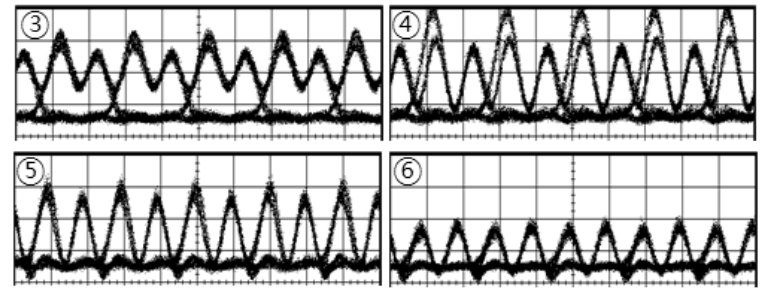

(d)

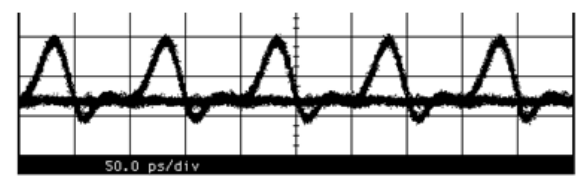

Fig. 3. Measured eye diagrams for (a) the input RZ signal, (b) the RZ-to-NRZ converted signal, (c) the 2RZ signals according to the change of the center wavelength of the OTGF (when (1) $1549.7 \mathrm{~nm}$, 
have shown that the RZ-to-NRZ method, in spite of any distortion of the converted NRZ signal, shows a better performance over the conventional NRZ modulation method. As mentioned before, the converted NRZ signal contains two red-chirped RZ components. In addition to the red chirping, generally, a blue chirping is also generated at the falling edge of the input pulse signal, but the red-chirping is much larger than the blue-chirping, when the driving current of SOA is relatively low [16]. With the same SOA used in this experiment, the previous paper [17] has shown the spectra of red-chirping by self-phase modulation (SPM) and the wavelength shift $\Delta \lambda$ was $0.51 \mathrm{~nm}$. Even though the current research involves a cross-phase modulation (XPM) of the SOA in a fiber loop mirror, the red-chirping is expected to be similarly generated.

The red-chirped components can be selected by optical narrowbandwidth grating filter TOGF (which has a bandwidth of $0.25 \mathrm{~nm}$ at $-3 \mathrm{~dB}$, JDS FITEL TB9226) and they will be 2RZ signal. Fig. 3 (c)-1) (6) shows several outputs $z^{\prime}(t)$, obtained by tuning the central wavelength of the TOGF. It clearly shows that the two RZ components (2RZ) can be extracted when the central wavelength is $1550.4 \mathrm{~nm}$ (Fig. 3 (c)-5). Therefore, the wavelength shift by the chirping was estimated to be $\sim 0.4 \mathrm{~nm}$. By removing one of the two RZ signals by the Mod2 in Fig. 1, the reconverted RZ signal $z(t)$ was finally achieved as shown in Fig. 3 (d). However, the pulse width of the reconverted RZ signal increased to $20 \mathrm{ps,}$ whereas, that of the original input RZ signal $x(t)$ was $6 \mathrm{ps}$. A $20 \mathrm{GHz}$ sampling oscilloscope (Agilent $86112 \mathrm{~A}$ module) and a $40 \mathrm{GHz} \mathrm{O} / \mathrm{E}$ converter (New Focus model-1004 $40 \mathrm{GHz}$ photodetector) in the experiment are used. The large undershoot observed in Fig. 3 (a) is due to an excessive ringing at the falling edge of RZ signal, whereas, similar ringing at the rising edge is hidden by the amplitude of RZ pulse.

As known in Fig. 3 (a) and (d), the RZ pulse of $6 \mathrm{ps}$ width shows a larger undershoot than that of $20 \mathrm{ps}$ width because of the limited bandwidth of the oscilloscope and $\mathrm{O} / \mathrm{E}$ converter. The exact width of the RZ pulses was measured with an optical autocorrelation equipment.

The BERs for the input RZ signal, the RZ-to-NRZ converted signal, and the NRZ-to-RZ reconverted signal are shown in Fig. 4. While the optical power penalty of the RZ-to-NRZ conversion was $1.64 \mathrm{~dB}$, the proposed NRZ-to-RZ conversion had a power penalty of $-1.4 \mathrm{~dB}$ to the injected NRZ signal. A negative power penalty signifies an increase in the receiver sensitivity, which is only possible due to a signal format conversion from NRZ to RZ. When compared to the original input RZ signal, the power penalty of the proposed conversion becomes $0.6 \mathrm{~dB}$.

Cascading of different types of networks, while maintaining an optical transparency, requires a reconversion to the original data format $(\mathrm{RZ} \rightarrow \mathrm{NRZ} \rightarrow \mathrm{RZ}$ in this paper). However, in this regard, it is not necessary to restore the original pulse width of the RZ signal. The reconversion depends only on the speed of the OTDM network at the final stage. Let three different networks are connected, say, a $100 \mathrm{~Gb} / \mathrm{s}-\mathrm{OTDM}$ (the bit period $T$ is $10 \mathrm{ps}$ ) network to a $10 \mathrm{~Gb} / \mathrm{s}-W D M(T=100 \mathrm{ps})$ network, which in turn connected to a $40 \mathrm{~Gb} / \mathrm{s}-\mathrm{OTDM}$ ( $T=25 \mathrm{ps}$ ) network, as shown in Fig. 5. The RZ-to-NRZ conversion (RZ of 6 ps pulse width is converted to NRZ of 100 ps width) and NRZ-to-RZ reconversion (NRZ of 100 ps width is converted to RZ of $20 \mathrm{ps}$ pulse width) as demonstrated in this paper, can 


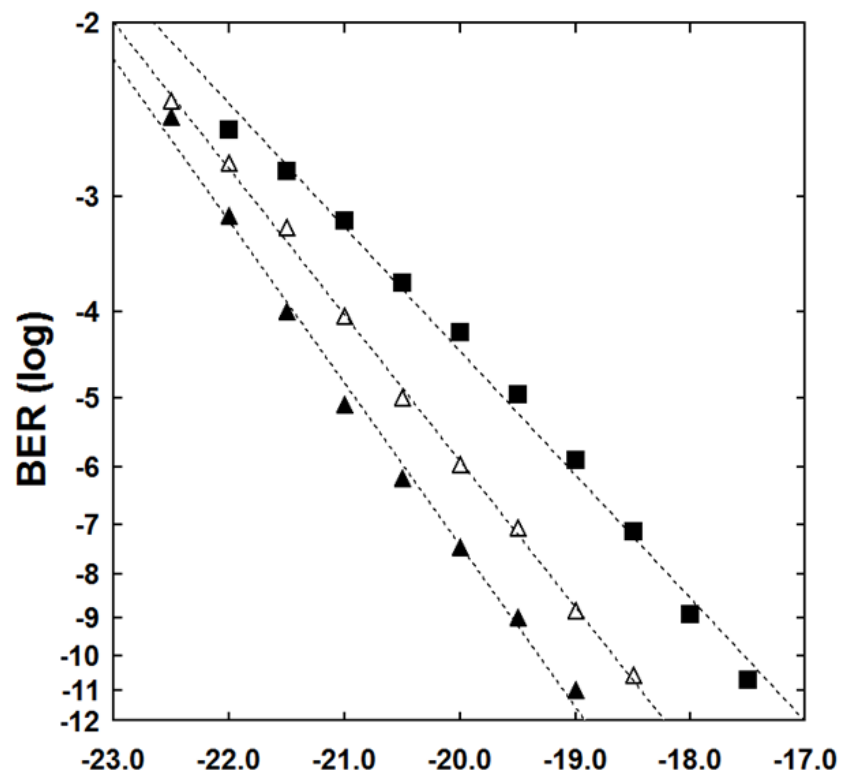

\section{Measured Received Power (dBm)}

Fig. 4. Measured BER curves for the RZ input signal ( $\mathbf{A})$, the RZ-to-NRZ converted signal ( $\mathbf{\square}$ ) and the NRZto-RZ converted signal $(\triangle)$.

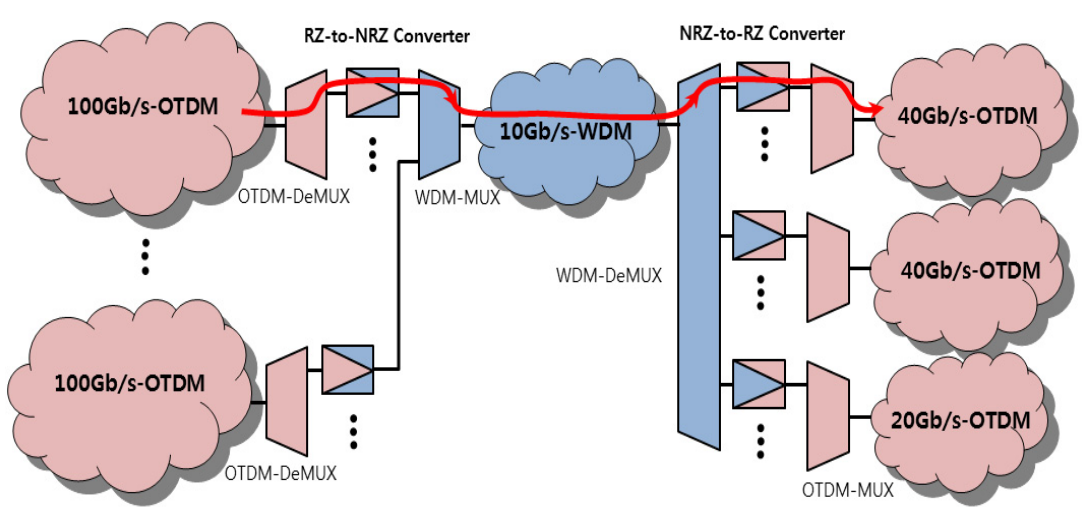

Fig. 5. One example of networks that can be supported by the RZ-to-NRZ conversion and NRZ-to-RZ reconversion as demonstrated in this paper.

support such network construction. On the other hand, if the speed of the last OTDM network is $100 \mathrm{~Gb} / \mathrm{s}$ or higher, the pulse compression method, as in Ref. [14], has to be used.

In the previous paper [5, 6], conversion of RZ to NRZ data allowed a 10$\mathrm{Gb} / \mathrm{s}$ error-free transmission up to $78 \mathrm{~km}$ through a standard single-mode fiber. Comparison of the conventional NRZ transmission according to the Mach-Zehnder modulation scheme with the proposed RZ-to-NRZ conversion, the latter shows a much improved transmission performance. This is primarily because the red-chirping $\left(\lambda_{0}+\Delta \lambda\right)$ at the rising and falling edges of the converted NRZ signal, travels slower than the unchirped components $\left(\lambda_{0}\right)$ at the center of the NRZ signal. Therefore, the two red-chirped components $\left(\lambda_{0}+\Delta \lambda\right)$ help in maintaining the shape of the NRZ signal, even though the RZ-to-NRZ converted signal is somewhat distorted. Moreover, the red-chirping components might give a pulse compression 
effect to the hidden RZ signal in the distorted NRZ signal during transmission. To restore back to the original NRZ signal, a dispersion compensated fiber (DCF) and/or a tunable dispersion compensator might be necessary. The performance of the NRZ-to-RZ reconversion after propagating through a dispersive fiber would be reported in the future paper.

\section{Conclusions}

A new all-optical NRZ-to-RZ data format reconversion has been demonstrated experimentally, using a simple scheme that consists of an optical modulator and an optical grating filter without the use of any additional optical clock. This was feasible because of the red-chirped NRZ signal formed by the previously proposed RZ-to-NRZ converter, using an SOAloop-mirror. The reconverted RZ signal had a pulse width of $\sim 20 \mathrm{ps}$, whereas, the original input RZ was $\sim 6$ ps. To restore the pulse width, a pulse compression, as reported in [14], may be needed. A polarization sensitive LiNbO3 optical Mach-Zehnder modulator was used to remove one of the 2RZ signals, thereby requiring a polarization controller (PC5 in the experimental setup). By replacing it with a polarization-insensitive optical modulator as in [15], the polarization problem can be solved. The proposed NRZ-to-RZ reconversion penalty was found to be $0.6 \mathrm{~dB}$ compared to that of the input RZ signal, whereas, the optical power penalty for RZ-to-NRZ conversion was $1.64 \mathrm{~dB}$. The proposed RZ-to-NRZ reconversion method will serve as a key block in linking and interfacing all-optical OTDM and WDM networks.

\section{Acknowledgments}

This work was supported by Kyungnam University Research Fund, 2010. 